\title{
The Zero Temperature Phase Diagram of the Kitaev Model
}

\author{
Charles Nash* \\ Department of Mathematical Physics, NUIM, Maynooth, Kildare, Ireland. \\ Denjoe O’Connor $\oplus$ \\ School of Theoretical Physics, DIAS, 10 Burlington Road, Dublin 4, Ireland.
}

\begin{abstract}
We show that the zero temperature phase diagram of the vortex free sector of the Kitaev model is in one to one correspondence with that of the classical dimer model on the corresponding lattice. We find that the model generically has three distinct phases. On a honeycomb lattice with a $3 \times 3$ fundamental domain all three phases are accessible. As the couplings are varied there are two distinct transitions. The new transition is one to a gapped phase that opens up in the interior of the $B$ phase of the Kitaev model.
\end{abstract}

Kitaev's observation [1] that a spin 1/2 system on a honeycomb lattice has a gapless phase with vortex excitations that obey non-Abelian statistics has stirred a lot of interest [2, 3, 4, 5, 6, 7, 8, 9]. Yao and Kivelson [5] extended Kitaev's considerations to the Fisher (triangle-honeycomb) lattice and found that its zero-temperature ground state is a chiral spin liquid. We extend these considerations to the more general setting.

The principal result of this note is:

- The vortex free zero temperature phases of Kitaev models are in one to one correspondence with the phases of classical dimer models.

A consequence of this is that just as with dimer models, Kitaev models divide into two classes: those defined on bipartite and non-bipartite lattices. A lattice is bipartite if its sites can be coloured black and white so that adjacent sites are always of the opposite colour. A Kitaev model is then bipartite if its Hamiltonian has no interaction between bonds of a given colour.

- Bipartite models have generically three phases, the third being a previously unnoticed gapped phase which we will refer to as the $C$ phase. This arises when a sufficiently large fundamental domain is considered, e.g. a $3 \times 3$ hexagonal domain.

As the couplings are varied the $C$ phase opens up in the interior of the $B$ phase and corresponds to giving a Dirac mass to the pair of degenerate massless Majorana Fermions of the $B$ phase. Close to the transition when the mass gap is small the model is well approximated by a continuum massive Dirac field theory.

- Non-bipartite couplings lift the degeneracy of the Majorana modes and the mass gap of one of the Majorana fields can go to zero to give a chiral spin liquid as discussed in [5].

The couplings of the non-bipartite model on the Fisher lattice can be choosen to correspond to those of a classical Ising model in its dimer realization [10]. At the Ising critical point the model has a low-lying massless spectrum that corresponds to a "relativistic" chiral Fermion [11] which becomes massive as the couplings are varied.

* cnash@thphys.nuim.ie

denjoe@stp.dias.ie 
The general situation is then that bipartite couplings can give rise to a Dirac mass for the Fermions while non-bipartite couplings give rise to a Majorana mass and lift the degeneracy of the two Majorana masses that comprise the Dirac Fermion of bipartite models.

The Kitaev spin model on the hexagonal lattice is given by the Hamiltonian

$$
\mathcal{H}=\sum_{x-\text { link }} J_{x} \sigma_{i}^{x} \sigma_{j}^{x}+\sum_{y-\text { link }} J_{y} \sigma_{i}^{y} \sigma_{j}^{y}+\sum_{z-\text { link }} J_{z} \sigma_{i}^{z} \sigma_{j}^{z}
$$

and partition function

$$
Z=\operatorname{Tr}\left(\mathrm{e}^{-\beta \mathcal{H}}\right)
$$

For simplicity we only consider the case of open boundary conditions as our focus is on the phase diagram of such models.

With open boundary conditions the model (1) can be rewritten, after a Jordan-Wigner transformation [1, 3, 5], in terms of Majorana operators $c_{i}$ with the partition function (2) given by

$$
Z=\sum_{u} \mathrm{e}^{-\beta H(u)}
$$

and the Hamiltonian

$$
H(u)=\frac{i}{2} \sum_{i j} c_{i} K_{i j}(u) c_{j}
$$

where $\left\{c_{i}, c_{j}\right\}=2 \delta_{i j}$. The matrix element $K_{i j}$ between site $i$ and $j$ is given by one of the couplings $J_{x}, J_{y}$ or $J_{z}$, up to an overall sign. This rewriting is valid for quite general couplings which can vary throughout the lattice. Yao and Kivelson [5] extended Kitaev's construction to the Fisher lattice (or triangle-honeycomb lattice).

The most general Kitaev model then assigns positive couplings to all links and a $Z_{2}$ gauge field $u_{i j}$ to each $z$-link between sites $i$ and $j$.

Unfortunately, for general temperatures the Kitaev model has not yet yielded an exact solution. However, the zero temperature ground state is known, thanks to Lieb's theorem [12], to be the vortex free state.

In the vortex free case the $u_{i j}$ become $u_{i j}^{\text {std }}$, and $K_{i j}$ is the Kasteleyn matrix of a classical dimer model. In this context $u_{i j}^{\text {std }}$ is a Kasteleyn orientation given by the "clockwise odd rule" [10, 13] around a plaquette. In the thermodynamic limit the eigenvalues of $K$ can be used to map out the phase diagram of the model. The phases of the zero temperature Kitaev model are therefore in one to one correspondence with those of classical dimer models.

Dimer models divide into two classes: those defined on bipartite and non-bipartite lattices. The hexagonal lattice is bipartite while the Fisher lattice is non-bipartite. The physics of the bipartite and non-bipartite lattices are quite distinct so we will discuss them separately.

We begin with the bipartite case for which one can write $K$ in the form

$$
K=\left(\begin{array}{cc}
0 & A \\
-A^{T} & 0
\end{array}\right)
$$

where the matrix $A$ has real entries and maps between the black and white links.

Fourier transforming $K$ one obtains a $2 d \times 2 d$ matrix where $d$ is the number of sites of the same colour in a fundamental domain.

The determinant of $A$ then gives a (Laurent) polynomial $P\left(\mathrm{e}^{i \theta}, \mathrm{e}^{i \phi}\right)$ in two unimodular complex variables. One can move off the unit circle by using some of the couplings to define general complex 
variables $z$ and $w$. We will refer the resulting polynomial as the spectral polynomial of the model. It can generically be written in the form

$$
P(z, w)=\sum_{a, b}(-1)^{a+b+a b} p_{a b} z^{a} w^{b}
$$

where $a$ and $b$ are integers and the coefficients $p_{a b}$ are positive real numbers determined by the couplings of the model.

The zero locus,

$$
P(z, w)=0,
$$

describes the gapless phase. This zero locus is referred to as the spectral curve.

Kenyon and Okounkov [14, 15] proved that the spectral curve (7), of a $d \times d$ fundamental domain with generic couplings is a Harnack curve and furthermore that every Harnack curve arises as the spectral curve of some bipartite dimer model. Such curves arose in the early twentieth century mathematics literature. They have very special properties and can be characterized by what is referred to as the amoeba of the curve.

Given the correspondence of the quadratic form of the vortex free sector of the Kitaev model to the Kasteleyn matrix of a classical dimer model, as pointed out above, eqn. (7), determines the phase diagram of the zero vortex sector of the Kitaev model. This phase diagram can conveniently be plotted in terms of the amoeba of eqn. (7) as we now describe.

Put simply, with $z=\mathrm{e}^{x+i \theta}$ and $w=\mathrm{e}^{y+i \phi}$, eqn. (17) can be solved for the angles $\theta$ and $\phi$ (see [16]). There are only two solutions $(\Theta(x, y), \Phi(x, y))$ and $(-\Theta(x, y),-\Phi(x, y))$ corresponding to $(z, w)$ and $(\bar{z}, \bar{w})$. At the boundary of the zero locus these angles become either 0 or $\pi$. The interior of the domain in the $x, y$ plane bounded by these curves is the amoeba.

In brief the amoeba can be plotted by noting that on it

$$
\prod_{p, q=0}^{1} P\left(\mathrm{e}^{x+i p \pi}, \mathrm{e}^{y+i q \pi}\right) \leq 0 .
$$

The amoeba has bounding curves called ovals. Ovals never intersect and can be both compact and non-compact. The genus, $g$, of the spectral curve is equal to the number of compact ovals.

The phase diagram of both classical bipartite dimer models and the zero temperature, zero vortex sector of Kitaev models then consists of the amoeba and its complement in the plane. The phase transitions of these models occur on the boundary of the amoeba, i.e. on the ovals.

For the $1 \times 1$ fundamental domain with positive couplings $J_{x}, J_{y}$ and $J_{z}$ the spectral curve is given by

$$
P(z, w)=1-1 / z-w=0
$$

where $z=\left(J_{z} / J_{x}\right) \mathrm{e}^{i \theta}$ and $w=\left(J_{y} / J_{z}\right) \mathrm{e}^{i \phi}$. We show the fundamental tile and its corresponding amoeba in Fig. 1, see [16] for more details on the amoeba in this case.

The complement of the amoeba consists of both compact and non-compact regions. In the terminology of dimer models, as models of melting crystals, the non-compact regions exterior to the bounding ovals constitute the frozen regions. The amoeba itself is referred to as the liquid phase, and the interior of the compact ovals as the gaseous phase.

The amoeba corresponds to the critical surface and gives the gapless $B$ phase of the Kitaev model. The frozen dimer regions go over to the Kitaev $A$ phase. There is however a third $C$ phase corresponding to the gaseous phase of the dimer model. 


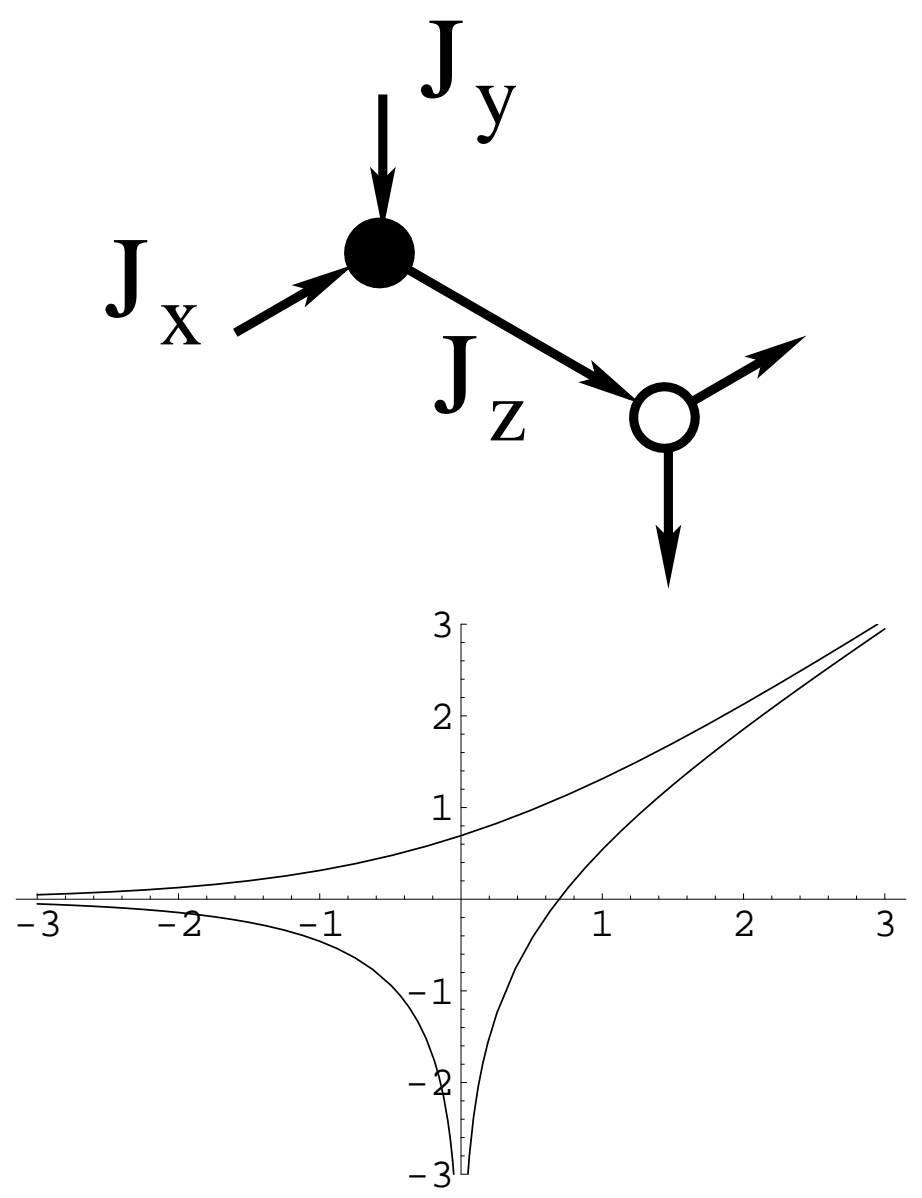

FIG. 1: On the left is the basic tile for the hexagonal lattice, showing the activities and our choice of Kasteleyn orientation with the corresponding amoeba on the right. The regions exterior to the curves shown corresponds to the Kitaev $A$ phase while the interior or amoeba gives the $B$ phase.

This $C$ phase seems not to have been observed in the literature. It is a novel phase in that it has a gap which corresponds to a Dirac mass for the pair of Majorana Fermions that describe the low energy excitations of the model.

The simplest example of a model that exhibits a $C$ phase is the square lattice as shown in Fig. 3. This can be obtained from the $3 \times 3$ hexagonal lattice by bond contractions.

The Kasteleyn matrix takes the form (5) and for the square lattice with four site fundamental tile, which is the simplest model with a compact oval, we have

$$
A=\left(\begin{array}{cc}
J_{11}-J_{11 x} \mathrm{e}^{-i \theta} & J_{12}-J_{12 y} \mathrm{e}^{-i \phi} \\
-J_{21}+J_{21 y} \mathrm{e}^{i \phi} & J_{22}-J_{22 x} \mathrm{e}^{i \theta}
\end{array}\right)
$$

The determinant of $A$ gives

$$
\begin{aligned}
P^{\text {Square }}= & J_{11} J_{22}+J_{11 x} J_{22 x}+J_{12} J_{21}+J_{12 y} J_{21 y} \\
& -J_{11} J_{22 x} \mathrm{e}^{i \theta}-\frac{J_{22} J_{11 x}}{\mathrm{e}^{i \theta}}-J_{12} J_{21 y} \mathrm{e}^{i \phi}-\frac{J_{21} J_{12 y}}{\mathrm{e}^{i \phi}}
\end{aligned}
$$

which can be parameterized in the form

$$
P^{\text {Square }}=D-A\left(z+\frac{1}{z}\right)-B\left(w+\frac{1}{w}\right)
$$




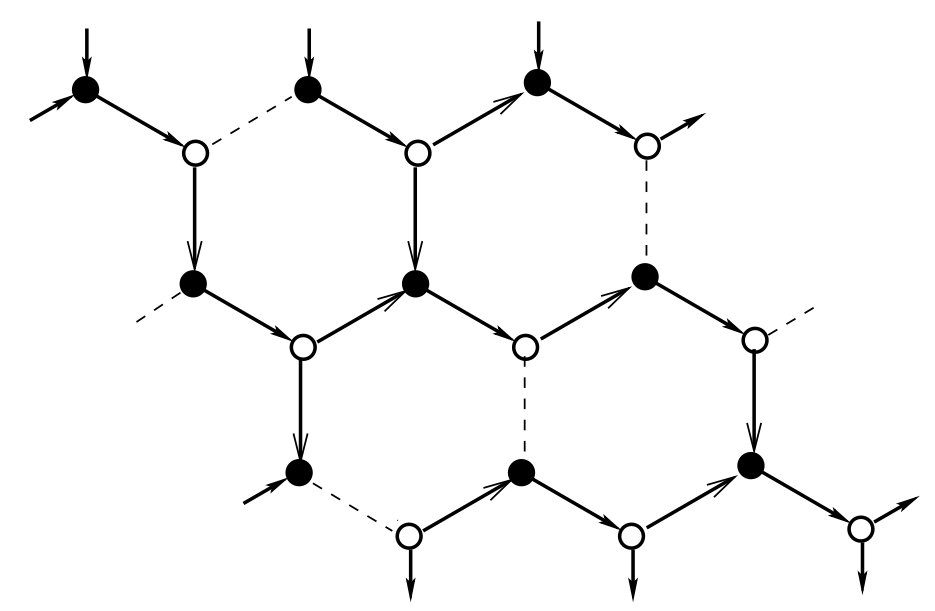

FIG. 2: The hexagonal tiling which reduces to the square lattice on bond contraction. Dashed lines correspond to bonds that are set to zero all other bonds are allowed to have generic values.
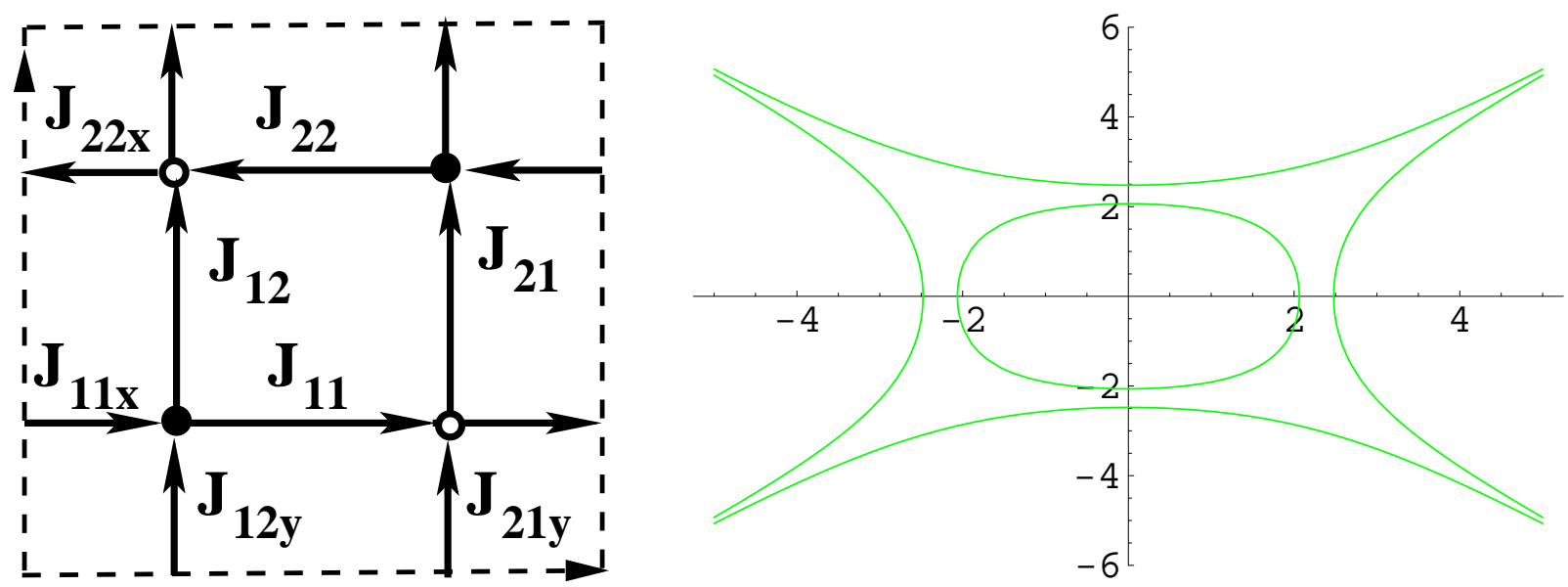

FIG. 3: The most general square lattice tiling with two vertices of each colour per tile and its amoeba with $t_{z}=t_{w}=0$ and $A=B=1$ and $D=10$ in eqn. (12).

where $D=2 A \cosh \left(t_{x}\right)+2 B \cosh \left(t_{y}\right)$ and $z=\mathrm{e}^{x+i \theta}$ and $w=\mathrm{e}^{y+i \phi}$ so that $z$ and $w$ can now take any complex values. The key point here is that this particular model has three phases: Those described in Kitaev's paper [1] as the $A$ and $B$ phases together with a new $C$ phase involving a mass gap. This phase arises when $t_{x}$ or $t_{y}$ is greater than zero. The phase is bounded by the curve $P^{\text {Square }}\left(\mathrm{e}^{x}, \mathrm{e}^{y}\right)=0$ which is the compact oval.

The general bipartite lattice - all of which can be obtained by special reductions, involving bond contractions [10, 15], of the hexagonal lattice with $d \times d$ fundamental domain so that there are $d^{2}$ vertices of each colour - is as follows: The phase diagram of the zero temperature, zero vortex Kitaev model is specified by the amoeba of the spectral polynomial which in turn is given as the determinant of $A$ as in (11). The Kasteleyn matrix, $K$, then describes a set of Majorana Fermions. On the amoeba two of these become massless or gapless. As one moves inside a compact oval these in turn acquire a mass, so that the system becomes gapped.

There will in fact be $g=(d-1)(d-2) / 2$ compact ovals. The number, $g$, of compact ovals is a topological invariant and is the genus of the spectral curve. As the couplings are varied some of 
the ovals may contract to points.

When bond couplings are set to zero, bounding phases will disappear. One can remove the corresponding bond from the tiling. However, the resulting lattice now has a flux through the double tile. One can remove this flux by altering the Kasteleyn orientation.

When the system is wrapped on a torus there is an additional term in the Hamiltonian [5] and a projection operator in the expression for the partition function (2). However, one can make the following observation: The spectrum of the Kasteleyn matrix, $K$, is discrete and on the amoeba the partition function depends on the shape parameter, $\tau$, (often referred to as the modular parameter) of the torus. As one approaches the non-compact boundary of the amoeba the torus becomes degenerate and there is a topological transition where the volume of the torus goes to zero [16]. This can best be seen in the context of classical dimers where the finite size corrections correspond to the conformal field theory of a massless Dirac Fermion.

The classical dimer model is also useful in gaining an understanding of the Kitaev model on a non-bipartite lattice. Again the minimum energy configuration will correspond to $u^{\text {std }}$ being a Kasteleyn orientation and $K(u)$ being a Kasteleyn matrix. The critical surface of the classical dimer model will describe the gapless phase.

A classic example of this situation is the dimer model on the Fisher lattice where the Pfaffian of $K$ gives the partition function of the two dimensional Ising model [10]. In the present context one can take over this result and state that the Kitaev model on the Fisher lattice will describe a single massive Majorana Fermion in the neighbourhood of the Ising critical surface. This will describe a gapped phase of the Kitaev model. The crossover from bipartite to non-bipartite Ising like behaviour was studied in the context of dimer models in [17].

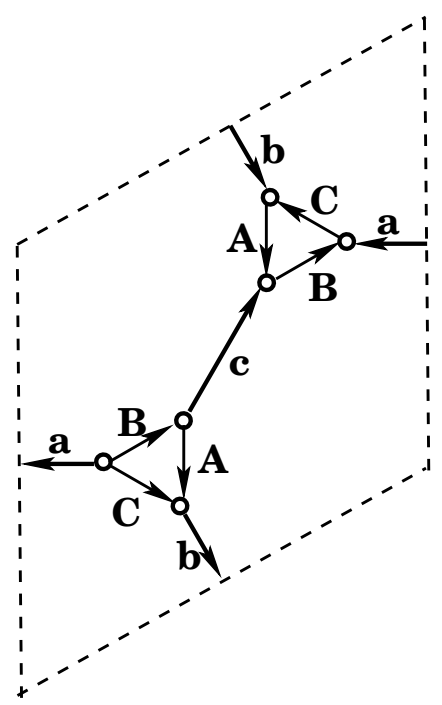

FIG. 4: The Fisher Lattice.

With any of $A, B$ or $C$ set to zero the Fisher lattice becomes bipartite. Setting $C=0$ gives the spectral polynomial

$$
P^{\text {Fisher }}=-a A^{2} \mathrm{e}^{i \theta}-b B^{2} \mathrm{e}^{i \phi}-a b c \mathrm{e}^{i(\theta+\phi)} .
$$

This is equivalent to the hexagonal lattice by a lattice reduction [10, 15]. 
The Kasteleyn matrix corresponding to the weights in Fig. [4 is given by

$$
K=\left(\begin{array}{cccccc}
0 & 0 & 0 & c & -B & A \\
0 & 0 & C & -B & -a \mathrm{e}^{i \theta} & 0 \\
0 & -C & 0 & A & 0 & -b \mathrm{e}^{i \phi} \\
-c & B & -A & 0 & 0 & 0 \\
B & a \mathrm{e}^{-i \theta} & 0 & 0 & 0 & C \\
-A & 0 & b \mathrm{e}^{-i \phi} & 0 & -C & 0
\end{array}\right)
$$

Setting $A=B=C=1$ and $a=1 / v_{1}, b=1 / v_{2}$ and $c=1 / v_{3}$, with $v_{i}=\tanh \left(k_{i}\right)$, the classical dimer model gives the partition function

$$
Z_{\text {Ising }}^{\mathrm{Hex}}=C_{\text {norm }} Z_{\text {dimer }}^{\text {Fisher }}\left(v_{1}^{-1}, v_{2}^{-1}, v_{3}^{-1}, 1,1,1\right)
$$

of the two dimensional Ising model on the hexagonal lattice. The normalization constant $C_{\text {norm }}$ is given by

$$
C_{\text {norm }}=2^{2 N M}\left(\sin \left(k_{1}\right) \sin \left(k_{2}\right) \sin \left(k_{3}\right)\right)^{N M}
$$

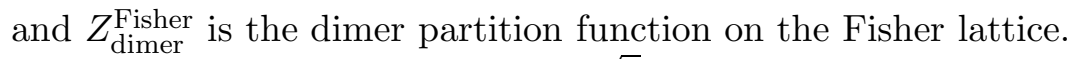

In the special case $a=b=c=\sqrt{3}$ with $A=B=C=1$, the model is gapless with two of the six eigenvalues zero at $\theta=\phi=\pi$. This is the critical point of the corresponding Ising model on the hexagonal lattice.

As a final observation we note that in the $B$ phase of the Kitaev model the system is described by a massless Dirac Fermion. Changing the parameters so that one crosses a compact oval gives this Fermion a mass. Adding non-bipartite couplings lifts the mass gap degeneracy of the two Majorana Fermions. The zero-temperature ground state is then a chiral spin liquid [5]. When the mass gap corresponding to the lightest Majorana Fermion is very small the zero vortex sector is well described by a relativistic chiral Fermion.

Acknowledgement This work was partly supported by EU-NCG Marie Curie Network No. MTRN-CT-2006-031962.

[1] A. Kitaev, "Anyons in an exactly solved model and beyond" Annals of Physics 321 (2006) 2, arXiv:cond-mat/0506438.

[2] J. K. Pachos, "The Wavefunction of an Anyon" Annals Phys. 322 (2007) 1254, arXiv:quant-ph/0605068.

[3] X.-Y. Feng, G.-M. Zhang, and T. Xiang, "Topological characterization of quantum phase transitions in a $S=1 / 2$ spin model" Phys. Rev. Lett. 98, (2007) 087204, arXiv:cond-mat/0610626.

[4] G. Kells, A. T. Bolukbasi, V. Lahtinen, J. K. Slingerland, J. K. Pachos and J. Vala, "Topological degeneracy and vortex manipulation in Kitaev's honeycomb model" Phys. Rev. Lett. to appear, arXiv:0804.2753.

[5] H. Yao and S. A. Kivelson, "Exact Chiral Spin Liquid with Non-Abelian Anyons" Phys. Rev. Lett. 99 (2007) 247203, arXiv:0708.0040.

[6] H.-D. Chen and Z. Nussinov, "Exact results of the Kitaev model on a hexagonal lattice: spin states, string and brane correlators, and anyonic excitations" J. Phys. A 41 (2008) 075001, arXiv:cond-mat/0703633.

[7] K.P. Schmidt, S. Dusuel and J. Vidal, "Emergent Fermions and Anyons in the Kitaev Model", Phys. Rev. Lett. 100, (2008) 057208, arXiv:0709.3017.

[8] S. Dusuel, K.P. Schmidt, J. Vidal, and R.L. Zaffino, "Perturbative Study of the Kitaev Model with Spontaneous Time-reversal Symmetry Breaking", Phys. Rev. B78, (2008) 125102, arXiv:0804.4775. 
[9] Dung-Hai Lee, Guang-Ming Zhang, and Tao Xiang, "Edge Solitons of Topological Insulators and Fractionalized Quasiparticles in Two Dimensions", Phys. Rev. Lett. 99, (2007) 196805, arXiv:0705.3499.

[10] M. E. Fisher, "On the Dimer Solution of Planar Ising Models", J. Math. Phys. 7 (1966) 1776.

[11] F. D. M. Haldane, "Model for a Quantum Hall Effect without Landau Levels: Condensed-Matter Realization of the "Parity Anomaly" ", Phys. Rev. Lett. 61, (1988) 2015.

[12] E. H. Lieb, "Flux Phase of the Half Filled Band", Phys. Rev. Lett. 73 (1994) 2158.

[13] P. W. Kasteleyn, "Graph Theory and Crystal Physics", in Graph Theory and Theoretical Physics pp.43-110, Academic Press, New York, 1967.

[14] R. Kenyon, A. Okounkov and S. Sheffield, "Dimers and Amoebae", Ann. Math. 163 (2006) 1019, arXiv:math-ph/0311005.

[15] R. Kenyon and A. Okounkov, "Planar dimers and Harnack curves", Duke Math. J. 131, (2006), 499, arXiv:math/0311062.

[16] C. Nash, D. O'Connor, "Topological Phase Transitions and Holonomies in the Dimer Model", J. Phys. A 42 (2009) 012002, arXiv:0809.2960.

[17] S. M. Bhattacharjee, "Crossover in an exactly solvable dimer model of domain walls with dislocations" Phys. Rev. Lett. 53, (1984) 1161. 